Article

\title{
On Maximal Elements with Applications to Abstract Economies, Fixed Point Theory and Eigenvector Problems
}

\author{
Liang-Ju Chu ${ }^{1}$ and Wei-Shih $\mathrm{Du}^{2, *(1)}$ \\ 1 Department of Mathematics, National Taiwan Normal University, Taipei 11677, Taiwan; \\ chulj@math.ntnu.eud.tw \\ 2 Department of Mathematics, National Kaohsiung Normal University, Kaohsiung 82444, Taiwan \\ * Correspondence: wsdu@mail.nknu.edu.tw
}

Received: 27 March 2019; Accepted: 10 June 2019; Published: 13 June 2019

\begin{abstract}
Two existence theorems of maximal elements in $H$-spaces are obtained without compactness. More accurately, we deal with the correspondence to be of $\mathcal{L}$-majorized mappings in the setting of noncompact strategy sets but merely requiring a milder coercive condition. As applications, we obtain an equilibrium existence theorem for general abstract economies, a new fixed point theorem, and give a sufficient condition for the existence of solutions of the eigenvector problem (EIVP).
\end{abstract}

Keywords: $H$-space; maximal element; $\mathcal{L}_{\varphi}$-majorized; abstract economy; eigenvector problem (EIVP)

\section{Introduction}

In the last fifty years, the classical Arrow-Debreu result [1] on the existence of Walrasian equilibria has been generalized in many directions. The existence of equilibrium in abstract economies with compact strategy sets in $R^{n}$ was proved in a seminar paper of Debreu [2], which extended the earlier work of Nash in game theory. Since then, the equilibrium existence theory for various models have been extensively studied by many authors. For example, there have been many generalizations of Debreu's theorem by Shafer and Sonnenschein [3], Borglin and Keiding [4], Gale and Mas-Colell [5], Yannelis and Prabhakar [6], Ding and Tan [7], Tarafdar [8], Yuan and Tarafdar [9], Park [10], He and Yannelis [11], and the references therein. In fact, Shafer and Sonnenschein [3] proved the equilibrium existence of abstract economies for the case where preferences may not be total or transitive. Gale et al. [5] gave in the same direction for competitive equilibria without ordered preferences. Borglin and Keiding [4] proved a new existence theorem for a compact abstract economy with KF-majorized preference correspondences. It is known that maximal element existence theorems are frequently used as the main tool for proving the existence of equilibria, e.g., see [10-21]. Furthermore, in 1983, Yannelis and Prabhakar [6] proved the existence of maximal elements with $\mathcal{L}$-majorized correspondences over compact subsets of topological linear spaces generalizing previous results.

In most of the known existence results of maximal elements and equilibria of abstract economies or general games, the convexity assumption plays a crucial role. In fact, the strategy sets of agents, the values of preference correspondences and constrained correspondences of agents may not have convexity structure. Hence, it is quite reasonable and valuable to study existence theorems in general nonconvex $H$-spaces. In addition, as in [12,16-18,22-24], most results on the existence theorems of maximal elements require the underlying spaces to be compact. However, as in many applications, maximal element existence theorems shall not need any compactness or paracompactness, such as the recent work by Ding, Kim and Tan [25], who proved some existence theorems of equilibria for noncompact abstract economies with $\mathcal{L}^{*}$-majorized preferences. The objective of this paper 
is to relax the compactness by some coercive conditions, and then use them to derive existence theorems of maximal elements, and establish several applications, containing general equilibria, fixed points, and eigenvectors. The rest of the paper is structured as follows: Section 2 contains some notation and definitions. In Section 3, we introduce some coercive conditions and prove two maximal element existence theorems to the mapping of class $\mathcal{L}$, and general $\mathcal{L}$-majorized mappings, respectively. Section 4 presents an equilibrium existence theorem for general abstract economies, and, as an application, we obtain a new fixed point theorem via our coercive condition. In Section 5, we give a sufficient condition for the existence of solutions of the eigenvector problem (EIVP). Finally, Section 6 contains some discussion and technical remarks.

\section{Materials and Methods}

The continuity property of set-valued mappings and the compactness of underlying spaces are crucial in solving many problems arising in mathematical analysis, and in particular the field of optimization theory. A new method to avoid the continuity can be found in He and Yannelis [11], who introduced the notion of "continuous inclusion property". Indeed, they proved new equilibrium existence results for games and economies with discontinuous and non-ordered preferences, together with some new fixed point theorems on compact convex and metrizable subsets in locally convex topological linear spaces. Their results shall be really different from our approach via coercive conditions on noncompact strategy sets. It should be noted that many underlying spaces are not compact in various economic situations, so it is important for us to encounter different types of preferences and obtain some existence results for such correspondences in noncompact settings. The mapping of class $\mathcal{L}$ and $\mathcal{L}$-majorized set-valued mappings were first introduced by Yannelis and Prabhakar [6] in 1983, which are useful for many applications in mathematical economics and usually need not be continuous in many situations. In this paper, we shall not require regular continuity of preferences and any compactness condition by involving various coercive conditions. Our motivation is to present a novel regularization of coercive conditions for a given $\mathcal{L}$-majorized set-valued mapping acting on general $\mathrm{H}$-spaces. Indeed, we shall introduce some coercive conditions and present two mathematical existence theorems of maximal elements, which constitute the main technical tools used to prove the existence results related to equilibria, fixed points, and eigenvectors. Those technical theorems are quite general and may be useful to a wide field of problems in economics. The following diagram shows the implications of the work:

H-space with covering polytopes + KKM theory + Coercive condition
$\Downarrow$
Existence of maximal elements

$\Downarrow$

Applications to equilibria, fixed points, and eigenvectors

We digest and list some definitions and notations as follows: for a nonempty set $X$, we denote the set of all subsets of $X$ by $2^{X}$, and the set of all nonempty finite subsets of $X$ by $\langle X\rangle$. For a set-valued mapping $T: X \longrightarrow 2^{Y}$, we say that a point $x \in X$ is a maximal element of $T$, if $T(x)=\varnothing$.

An $\boldsymbol{H}$-space is a topological space $X$, together with a family $\left\{\Gamma_{D}\right\}$ of some nonempty contractible subsets of $X$ indexed by $D \in\langle X\rangle$ such that $\Gamma_{D} \subset \Gamma_{D^{\prime}}$ whenever $D \subset D^{\prime}$. The notion of $H$-space was introduced in 1988 by Bardaro and Ceppitelli [22]. Since then, there have appeared numerous applications and generalizations in the literature $[8,16,18,19,23,25,26]$. Given an $H$-space $\left(X,\left\{\Gamma_{D}\right\}\right)$, a nonempty subset $C$ of $X$ is said to be $H$-convex if $\Gamma_{D} \subset C$ for all $D \in\langle C\rangle$. For a nonempty subset $C$ of $X$, we define the $H$-convex hull of $C$ as $H-c o C:=\bigcap\{W \mid W$ is $H$-convex in $X$ and $C \subset W\}$. Moreover, for any $D \in\langle X\rangle, H-c o D$ is called a polytope. We say that $X$ is an $H$-space with covering polytopes, 
if for any subset $C$ of $X$, and $y \in H-c o C$, there is a $D \in\langle C\rangle$ such that the polytope $H$-coD contains $y$. For example, a locally convex topological vector space $X$ is an $H$-space with covering polytopes, by setting $\Gamma_{D}=c o D$ for all $D \in\langle X\rangle$.

We will encounter different kinds of preferences in various economic situations as follows: Let $X$ and $Y$ be $H$-spaces, $T: X \longrightarrow 2^{Y}$ a set-valued mapping, and $\varphi: X \longrightarrow Y$ be a single-valued mapping.

(1) $T$ is said to be of class $\mathcal{L}_{\varphi}$, if

(a) for each $x \in X, \varphi(x) \notin H$-coT $(x)$,

(b) for each $y \in Y, T^{-1}(y)$ is open in $X$.

(2) A set-valued mapping $T_{x}: X \longrightarrow 2^{Y}$ is an $\mathcal{L}_{\varphi}$-majorant of $T$ at $x$, if there exists an open neighborhood $N_{x}$ of $x$ in $X$ such that

(a) for each $z \in X, T(z) \subset T_{x}(z)$,

(b) for each $z \in N_{x}, \varphi(z) \notin H-c o T_{x}(z)$,

(c) for each $y \in Y, T_{x}^{-1}(y)$ is open in $X$.

(3) $T$ is said to be $\mathcal{L}_{\varphi}$-majorized if, for each $x \in X$ with $T(x) \neq \varnothing$, there exists an $\mathcal{L}_{\varphi}$-majorant of $T$ at $x$.

Remark 1. It is worth mentioning that case $\varphi: X \longrightarrow X$ is the identity mapping on $X$, with $Y=X$; all notations above shall be simplified to be of class $\mathcal{L}, \mathcal{L}$-majorant, and $\mathcal{L}$-majorized, respectively. It should be noted that every set-valued mapping of class $\mathcal{L}$ is $\mathcal{L}$-majorized. However, $\mathcal{L}$-majorized mappings need not be of class $\mathcal{L}$, such as the set-valued mapping $T: X \longrightarrow 2^{X}$, where $X=[0,1)$, defined by

$$
T(z):= \begin{cases}\left\{y \in X \mid 0 \leq y \leq z^{2}\right\}, & \text { if } z \in(0,1), \\ \varnothing, & \text { if } z=0 .\end{cases}
$$

In fact, for any $x \in(0,1)$, let $N_{x}=X$ and define $T_{x}: X \longrightarrow 2^{X}$ by

$$
T_{x}(z):= \begin{cases}\{y \in X \mid 0 \leq y<z\}, & \text { if } z \in(0,1) . \\ \varnothing, & \text { if } z=0 .\end{cases}
$$

Then, $T_{x}$ is an $\mathcal{L}$-majorant of $T$ at $x$, and hence $T$ is $\mathcal{L}$-majorized. However, $T$ is not of class $\mathcal{L}$, since $T^{-1}(y)$ is not open for each $y \in(0,1)$.

\section{Results on Maximal Elements}

In this section, we shall develop two general existence theorems of maximal elements, so that we can apply them to abstract economies, together with a fixed point theorem, and eigenvector problems, respectively. In order to establish our main results, we need two fundamental lemmas. The following Lemma 1 is an extension of [6] (Lemma 5.1) in $H$-spaces.

Lemma 1. Let $X$ be a topological space and $Y$ be an $H$-space with covering polytopes. If $T: X \longrightarrow 2^{Y}$ is a set-valued mapping with open lower sections, then the set-valued mapping $H$-coT:X $\longrightarrow 2^{Y}$, defined by $H-\operatorname{co} T(x)=H-c o(T(x))$, has open lower sections.

Proof. For any $y_{0} \in Y$, either $(H-c o T)^{-1}\left(y_{0}\right)=\varnothing$ or $(H-c o T)^{-1}\left(y_{0}\right) \neq \varnothing$. In case $(H \text {-coT })^{-1}\left(y_{0}\right) \neq \varnothing$, we take any $x_{0} \in(H-c o T)^{-1}\left(y_{0}\right)$. Thus,

$$
y_{0} \in H-\operatorname{coT}\left(x_{0}\right)=H-\operatorname{co}\left(T\left(x_{0}\right)\right) .
$$


Since $Y$ is an $H$-space with covering polytopes, there is a finite subset $D$ of $T\left(x_{0}\right)$ such that the polytope $H-c o D$ contains $y_{0}$. Say, $D=\left\{y_{1}, y_{2}, \cdots, y_{n}\right\}$. Then, for each $i=1,2, \cdots, n$, we have $y_{i} \in D \subset T\left(x_{0}\right)$. It follows that $x_{0} \in T^{-1}\left(y_{i}\right)$ for all $i=1,2, \cdots, n$. Define $U=\bigcap_{i=1}^{n} T^{-1}\left(y_{i}\right)$. By assumption, each lower section $T^{-1}\left(y_{i}\right)$ is open, so that $U$ is an open set containing $x_{0}$. Now, for any $x \in U$, we have $x \in T^{-1}\left(y_{i}\right)$ for all $i=1,2, \cdots, n$, and hence $y_{i} \in T(x)$. It follows that

$$
y_{0} \in H-\operatorname{coD} \subset H-\operatorname{coT}(x) .
$$

Accordingly, $x \in(H-c o T)^{-1}\left(y_{0}\right)$. This yields that $U \subset(H-c o T)^{-1}\left(y_{0}\right)$, and therefore $(H-c o T)^{-1}\left(y_{0}\right)$ must be open. Thus, the proof is complete.

The following version on the KKM theorem is an extension of Ding and Tan [7], and some generalizations of $[10,13,27,28]$, due to Chang and Ma [13] (Theorem 1):

Lemma 2. Let (X, $\left.\left\{\Gamma_{D}\right\}\right)$ be an $H$-space. If $F(x)$ is a compactly closed (or open) subset of $X$ for all $x \in X$ such that $\Gamma_{A} \subset F(A)$ for each $A \in\langle X\rangle$, then the family $\{F(x) \mid x \in X\}$ has the finite intersection property.

We now establish our first existence theorem of maximal elements, which is a generalization of the earlier Fan version [29] (Theorem 4).

Theorem 1. Let $\left(X,\left\{\Gamma_{D}\right\}\right)$ be an $H$-space with covering polytopes, $C$ be a nonempty $H$-convex subset of $X$, and $T: C \longrightarrow 2^{X}$ be a set-valued mapping of class $\mathcal{L}$. If there exists a nonempty compact subset $K$ of $C$ such that $K \cap H-\operatorname{coT}(x) \neq \varnothing$ for all $x \in C \backslash K$, then $T$ has a maximal element in $C$.

Proof. Assume that $T(x) \neq \varnothing$ for all $x \in C$ so that $H$-coT(x) $\neq \varnothing$. Since $T$ is of class $\mathcal{L}$, it has open lower sections. By Lemma $1,(H-c o T)^{-1}(x)$ is open for all $x \in X$. Define a set-valued mapping $F: C \longrightarrow 2^{C}$ by $F(x):=C \backslash(H-c o T)^{-1}(x)$ for all $x \in C$. Then, each $F(x)$ is closed in $C$. Now, we claim that $\bigcap_{x \in K} F(x) \subset K$. In fact, if there is some $y \in \bigcap_{x \in K} F(x)$ but $y \notin K$, then

$$
y \in \bigcap_{x \in K} F(x)=\bigcap_{x \in K}\left(C \backslash(H-c o T)^{-1}(x)\right)=C \backslash \bigcup_{x \in K}(H-c o T)^{-1}(x) .
$$

It follows that $K \cap H-c o T(y)=\varnothing$, which contradicts with our assumption. Thus, $\bigcap_{x \in K} F(x)$ is a closed subset of the compact set $K$, so that $\bigcap_{x \in K} F(x)$ is also compact. Since $H$-coT $(x) \neq \varnothing$ for all $x \in C$, we can take a $y_{x} \in H-c o T(x)$, so that $x \in(H-c o T)^{-1}\left(y_{x}\right)$. This shows that $C=\bigcup_{x \in C}(H-c o T)^{-1}\left(y_{x}\right)$. Therefore,

$$
\bigcap_{x \in C} F(x)=\bigcap_{x \in C}\left(C \backslash(H-c o T)^{-1}(x)\right)=C \backslash \bigcup_{x \in C}(H-c o T)^{-1}(x)=\varnothing .
$$

This implies that $F$ cannot be a KKM-mapping by Lemma 2; that is, there exists a finite subset $A=\left\{x_{1}, x_{2}, \cdots, x_{n}\right\}$ of $C$ such that $\Gamma_{A} \not \subset F(A)$. Let $z \in \Gamma_{A} \backslash F(A)$. Since $C$ is $H$-convex and contains $A$, we have

$$
z \in \Gamma_{A} \backslash F(A) \subset C \backslash \bigcup_{i=1}^{n} F\left(x_{i}\right)=\bigcap_{i=1}^{n}(H-c o T)^{-1}\left(x_{i}\right) .
$$

Consequently, $z \in(H-c o T)^{-1}\left(x_{i}\right)$ for each $i=1,2, \cdots, n$. Equivalently, $x_{i} \in H$-coT $(z)$ for each $i=1,2, \cdots, n$. It follows that $z \in \Gamma_{A} \subset H$-co $A \subset H$-coT $(z)$, which contradicts the assumption that $T$ is of class $\mathcal{L}$. Thus, the proof is complete. 
Remark 2. Whenever $C$ is compact and H-convex, the coercive condition in Theorem 1 obviously holds by taking $K=C$. Therefore, Theorem 1 improves the result of [18] (Theorem 2). In particular, when the coercive condition becomes that "there exists a nonempty compact subset $K$ of $C$ and $y_{0} \in K$ such that $y_{0} \in H$-coT $(x)$ for all $x \in C \backslash K$," the mapping of class $\mathcal{L}$ can be relaxed as $\mathcal{L}$-majorized mappings. Next, the existence theorem deals with the case of $\mathcal{L}$-majorized mappings.

Theorem 2. Let $\left(X,\left\{\Gamma_{D}\right\}\right)$ be an $H$-space with covering polytopes, $C$ be a nonempty $H$-convex subset of $X$, and $T: C \longrightarrow 2^{C}$ be an $\mathcal{L}$-majorized set-valued mapping. If there exists a nonempty compact subset $K$ of $C$ and $y_{0} \in K$ such that $y_{0} \in H$-coT $(x)$ for all $x \in C \backslash K$, then $T$ has a maximal element in $C$.

Proof. Assume that $T(x) \neq \varnothing$ for all $x \in C$. Since $T$ is $\mathcal{L}$-majorized, for each $x \in C$, there exists an $\mathcal{L}$-majorant $T_{x}: C \longrightarrow 2^{C}$ and an open neighborhood $N_{x}$ of $x$ in $C$ such that

(a) $T(z) \subset T_{x}(z)$ for all $z \in X$;

(b) $z \notin H-c o T_{x}(z)$ for all $z \in N_{x}$;

(c) $T_{x}^{-1}(y)$ is open in $C$ for each $y \in C$.

Note that the family $\left\{N_{x} \mid x \in K\right\}$ forms an open covering of $K$. By the compactness of $K$, there is a finite subcover $\left\{N_{x_{i}} \mid i=1,2, \cdots, n\right\}$. Let $U=\bigcup_{i=1}^{n} N_{x_{i}}$. Then, $U$ is open in $C$ and $K \subset U \subset C$. Note that, by condition (c) and Lemma 1 , the set $\left(H-c^{-} T_{x_{i}}\right)^{-1}(y)$ is open for each $y \in C$. Now, we define $S: C \longrightarrow 2^{C}$ by

$$
S(z):= \begin{cases}\bigcap_{i=1}^{n} S_{i}(z), & \text { if } z \in U, \\ \left\{y_{0}\right\}, & \text { if } z \in C \backslash U,\end{cases}
$$

where $S_{i}: U \longrightarrow 2^{C}$ is the restriction of $H$-coT $x_{x_{i}}$ on $U$ for each $i=1,2, \cdots, n$. We claim that $S$ is a set-valued mapping of class $\mathcal{L}$. Indeed, for each $y \in C$, in case $y \neq y_{0}$, we have

$$
S_{i}^{-1}(y)=\left\{z \in U \mid y \in S_{i}(z)\right\}=\left\{z \in U \mid y \in H-\operatorname{coT}_{x_{i}}(z)\right\}=U \cap\left(H-\operatorname{coT}_{x_{i}}\right)^{-1}(y),
$$

which is open in $C$. Therefore, the set

$$
\begin{aligned}
S^{-1}(y) & =\{z \in C \mid y \in S(z)\} \\
& =\{z \in U \mid y \in S(z)\} \cup\{z \in C \backslash U \mid y \in S(z)\} \\
& =\{z \in U \mid y \in S(z)\} \\
& =\left\{z \in U \mid y \in \bigcap_{i=1}^{n} S_{i}(z)\right\} \\
& =U \cap\left(\bigcap_{i=1}^{n} S_{i}^{-1}(y)\right)
\end{aligned}
$$

is also open in $C$. In case $y=y_{0}$, by assumption and (a), for all $z \in C \backslash K$, we have

$$
y_{0} \in H-\operatorname{coT}(z) \subset H-\operatorname{coT}_{x_{i}}(z) \text { for each } i=1,2, \cdots, n .
$$


Thus, $C \backslash K \subset\left(H-c o T_{x_{i}}\right)^{-1}\left(y_{0}\right)$ so that $C \backslash U \subset\left(H-c o T_{x_{i}}\right)^{-1}\left(y_{0}\right)$ for each $i=1,2, \cdots, n$. Therefore,

$$
\begin{aligned}
S^{-1}\left(y_{0}\right) & =\left\{z \in C \mid y_{0} \in S(z)\right\} \\
& =\left\{z \in U \mid y_{0} \in S(z)\right\} \cup\left\{z \in C \backslash U \mid y_{0} \in S(z)\right\} \\
& =\left(U \cap\left(\bigcap_{i=1}^{n} S_{i}^{-1}\left(y_{0}\right)\right)\right) \cup(C \backslash U) \\
& =\left(U \cap\left(\bigcap_{i=1}^{n}\left(H-c o T_{x_{i}}\right)^{-1}\left(y_{0}\right)\right)\right) \cup(C \backslash U) \\
& =\left(\bigcap_{i=1}^{n}\left(H-c o T_{x_{i}}\right)^{-1}\left(y_{0}\right)\right) \cup(C \backslash U) \\
& =\bigcap_{i=1}^{n}\left(H-c o T_{x_{i}}\right)^{-1}\left(y_{0}\right),
\end{aligned}
$$

which is also open in $C$. Furthermore, for any $z \in C=U \cup(C \backslash U)$, in case $z \in U$, then $z \in N_{x_{i}}$ for some $i$, so that $z \notin H-\operatorname{coT} T_{x_{i}}(z)=S_{i}(z)$. Since $H-\cos (z) \subset H$-coT $x_{x_{i}}(z)=S_{i}(z)$, it follows that $z \notin H-\cos (z)$. On the other hand, if $z \in C \backslash U$, then $z \in C \backslash K$, and hence $z \neq y_{0}$. Therefore, $z \notin\left\{y_{0}\right\}=S(z)=H-\operatorname{coS}(z)$. From the above illustration, $S$ is a set-valued mapping of class $\mathcal{L}$.

Next, we want to show that $y_{0} \in H-\operatorname{coS}(z)$ for all $z \in C \backslash K$. In fact, we notice that $C \backslash K=$ $(C \backslash U) \cup(U \backslash K)$. If $z \in C \backslash U$, then $S(z)=\left\{y_{0}\right\}$, and hence $y_{0} \in\left\{y_{0}\right\}=H$-coS $(z)$. On the other hand, if $z \in U \backslash K$, we note that for each $i=1,2, \cdots, n$,

$$
T(z) \subset T_{x_{i}}(z) \subset S_{i}(z) \text { for all } z \in U .
$$

This yields that $T(z) \subset S(z)$ for all $z \in U$. According to our assumption, we have $y_{0} \in H$-coT $(z) \subset$ $H-\cos (z)$. The above information implies that the coercive hypothesis in Theorem 1 is satisfied by taking $C_{0}=\left\{y_{0}\right\}$. Thus, $S$ has a maximal element $\hat{x} \in C$; that is, $S(\hat{x})=\varnothing$. This implies that $\hat{x} \in U$ and $T(\hat{x})=\varnothing$, since $T(\hat{x}) \subset S(\hat{x})$, which is a contradiction. Therefore, we complete the proof.

\section{Results on Abstract Economies}

Let $I$ be any (finite or infinite) set of agents. For each $\alpha \in I$, let $C_{\alpha}$ be a strategy set in a topological space $X_{\alpha}$, and $C:=\prod_{\alpha \in I} C_{\alpha}$. An abstract economy is defined as a family of order quadrauples $\mathbf{\Omega}:=\left(C_{\alpha}, A_{\alpha}, B_{\alpha}, P_{\alpha}\right)_{\alpha \in I}$ such that, for each $\alpha \in I, A_{\alpha}, B_{\alpha}: C \longrightarrow 2^{C_{\alpha}}$ are constraint correspondences, and $P_{\alpha}: C \longrightarrow 2^{C_{\alpha}}$ is a preference correspondence. An equilibrium of $\Omega$ is a point $\hat{x} \in C$ such that, for each $\alpha \in I, \hat{x}_{\alpha}=\pi_{\alpha}(\hat{x}) \in \operatorname{clB}_{\alpha}(\hat{x})$ and $A_{\alpha}(\hat{x}) \cap P_{\alpha}(\hat{x})=\varnothing$, where $\pi_{\alpha}: C \longrightarrow C_{\alpha}$ denotes the projection mapping from $C$ onto $C_{\alpha}$. For more details on this, see Gale and Mas-Colell [5], and Shafer and Sonnenschein [3]. It is known that, if $\left(X_{\alpha}, \Gamma^{\alpha}{ }_{D_{\alpha}}\right)_{\alpha \in I}$ is a family of $H$-spaces, Tarafder [8,26] has shown that the product space $X=\prod_{\alpha \in I} X_{\alpha}$ with product topology is also an $H$-space, together with the family $\left\{\Gamma_{D} \mid D \in\langle X\rangle\right\}$, where $\Gamma_{D}$ is defined by $\Gamma_{D}=\prod_{\alpha \in I} \Gamma_{\pi_{\alpha}(D)}^{\alpha}$. In addition, the product of $H$-convex subsets is also $H$-convex. For two correspondences $S, T: C \longrightarrow 2^{C_{\alpha}}$, the correspondence $S \cap T: C \longrightarrow 2^{C_{\alpha}}$ is defined by $(S \cap T)(x)=S(x) \cap T(x)$ for each $x \in C$.

In this sequel, we shall prove an existence theorem of equilibria for the abstract economy $\Omega:=\left(C_{\alpha}, A_{\alpha}, B_{\alpha}, P_{\alpha}\right)_{\alpha \in I}$. We first establish a fundamental lemma as follows.

Lemma 3. Let $X_{\alpha}$ be an $H$-space with covering polytopes for each $\alpha \in I$, and $X=\prod_{\alpha \in I} X_{\alpha}$. Suppose that, for each $\alpha \in I$, the following assumptions are satisfied:

(1) $C_{\alpha}$ is an $H$-convex subset of $X_{\alpha}$, and $K_{\alpha}$ is a nonempty compact subset of $C_{\alpha}$;

(2) $P_{\alpha}: C \longrightarrow 2^{C_{\alpha}}$ is $\mathcal{L}_{\pi_{\alpha}}$-majorized, where $C:=\prod_{\alpha \in I} C_{\alpha}$; 
(3) the set $D_{\alpha}:=\left\{x \in C \mid P_{\alpha}(x) \neq \varnothing\right\}$ is open in $C$;

(4) there exists $y_{\alpha} \in K_{\alpha}$ such that $y_{\alpha} \in H$-co $P_{\alpha}(x)$ for all $x \in C \backslash K$, where $K:=\prod_{\alpha \in I} K_{\alpha}$.

Then, there exists an $\hat{x}$ such that $P_{\alpha}(\hat{x})=\varnothing$ for all $\alpha \in I$.

Proof. For each $x \in C$, we let $I(x):=\left\{\alpha \in I \mid P_{\alpha}(x) \neq \varnothing\right\}$. Note that, if $I(x)=\varnothing$, then $P_{\alpha}(x)=\varnothing$ for all $\alpha \in I$. Therefore, this leads us to define a set-valued mapping $T: C \longrightarrow 2^{C}$ by

$$
T(x):= \begin{cases}\bigcap_{\alpha \in I(x)} S_{\alpha}(x), & \text { if } I(x) \neq \varnothing, \\ \varnothing, & \text { if } I(x)=\varnothing,\end{cases}
$$

where $S_{\alpha}(x):=\prod_{j \in I, j \neq \alpha} C_{j} \otimes P_{\alpha}(x)=\left\{\left(x_{j}\right)_{l \in I} \mid x_{j} \in C_{j}\right.$ for $j \neq \alpha$ and $\left.x_{\alpha} \in P_{\alpha}(x)\right\}$ for each $x \in C$. We claim that $T$ satisfies all hypotheses and the coercive condition in Theorem 2 . First, we note that $K:=\prod_{\alpha \in I} K_{\alpha}$ is a compact subset of $C$, since each $K_{\alpha}$ is compact. Next, for each $x \in C$ with $I(x) \neq \varnothing$, we have $S_{\alpha}(x) \neq \varnothing$ for all $\alpha \in I(x)$. By assumption (2), for each fixed $\alpha \in I(x)$, there exists an $\mathcal{L}_{\pi_{\alpha}}$-majorant $T_{\alpha}$ of $P_{\alpha}$ at $x$ and an open neighborhood $N_{\alpha}$ of $x$ in $C$ such that

(a) $P_{\alpha}(z) \subset T_{\alpha}(z)$ for each $z \in C$;

(b) $\pi_{\alpha}(z) \notin H-\cot T_{\alpha}(z)$ for each $z \in N_{\alpha}$;

(c) $T_{\alpha}^{-1}(y)$ is open in $C$ for all $y \in C_{\alpha}$.

By assumption (3), we may assume that $N_{\alpha} \subset D_{\alpha}$, so that $P_{\alpha}(z) \neq \varnothing$ for all $z \in N_{\alpha}$. Now, we define $T_{x}: C \longrightarrow 2^{C}$ by $T_{x}(z)=\pi_{\alpha}^{-1}\left(T_{\alpha}(z)\right)$ for $z \in C$. Then, we can show that $T_{x}$ is an $\mathcal{L}$-majorant of $T$ at $x$. Indeed, for each $z \in C$, if $y \in T(z)$, then $y \in \bigcap_{\alpha \in I(z)} S_{\alpha}(z)=\bigcap_{\alpha \in I(z)} \prod_{j \in I, j \neq \alpha} C_{j} \otimes P_{\alpha}(z)$. It follows that $\pi_{\alpha}(y) \in P_{\alpha}(z) \subset T_{\alpha}(z)$ by (a). Thus, $y \in \pi_{\alpha}^{-1}\left(T_{\alpha}(z)\right)=T_{x}(z)$, and so $T(z) \subset T_{x}(z)$ for all $z \in C$. Next, for each $z \in N_{\alpha}$, by (b), $\pi_{\alpha}(z) \notin H$-co $T_{\alpha}(z)$, and hence $z \notin \pi_{\alpha}^{-1}\left(H-c o T_{\alpha}(z)\right)$. Since $H$-co $T_{x}(z) \subset \pi_{\alpha}^{-1}\left(H-c o T_{\alpha}(z)\right)$, it follows that $z \notin H$-co $T_{x}(z)$. Furthermore, for each $y \in C$, we have $T_{x}^{-1}(y)=T_{\alpha}^{-1}\left(\pi_{\alpha}(y)\right)$, which is open in $C$ by (c). Thus, $T_{x}$ is an $\mathcal{L}$-majorant of $T$ at $x$, and hence $T$ is $\mathcal{L}$-majorized. In addition, by assumption (4), we can take $y:=\left(y_{\alpha}\right) \in K$, so that $y \in H$-coT(x) for all $x \in C \backslash K$. It follows from Theorem 2 that there is a maximal point $\hat{x}$ of $T$; that is, $T(\hat{x})=\varnothing$, which implies $P_{\alpha}(\hat{x})=\varnothing$ for all $\alpha \in I$.

Next, we use the above Lemma to establish our main existence theorem, which improves previous results of Yuan and Tarafdar [9] (Theorem 4.1) and Kim [24] (Theorem 3), and answers a question posed by Yannelis and Prabhakar [6].

Theorem 3. Let $\Omega:=\left(C_{\alpha}, A_{\alpha}, B_{\alpha}, P_{\alpha}\right)_{\alpha \in I}$ be an abstract economy, where $C_{\alpha}$ is an H-convex subset of an $H$-space $X_{\alpha}$ with covering polytopes for each $\alpha \in I$. Suppose that, for each $\alpha \in I$, there is a nonempty compact subset $K_{\alpha}$ of $C_{\alpha}$ such that

(1) $A_{\alpha}(x) \neq \varnothing$ and $H$-co $A_{\alpha}(x) \subset B_{\alpha}(x)$ for each $x \in C$, where $C:=\prod_{\alpha \in I} C_{\alpha}$;

(2) the mapping $A_{\alpha}: C \longrightarrow 2^{C_{\alpha}}$ has open lower sections;

(3) the mapping $c l B_{\alpha}: C \longrightarrow 2^{C_{\alpha}}$ is upper semicontinuous;

(4) the mapping $A_{\alpha} \cap P_{\alpha}: C \longrightarrow 2^{C_{\alpha}}$ is $\mathcal{L}_{\pi_{\alpha}}$-majorized;

(5) the set $D_{\alpha}:=\left\{x \in C \mid A_{\alpha}(x) \cap P_{\alpha}(x) \neq \varnothing\right\}$ is open in $C$;

(6) there exists $y_{\alpha} \in K_{\alpha}$ such that $y_{\alpha} \in H$-co $\left(A_{\alpha} \cap P_{\alpha}\right)(x)$ for all $x \in C \backslash K$, where $K:=\prod_{\alpha \in I} K_{\alpha}$.

Then, there exists an equilibrium of $\Omega$.

Proof. For each $\alpha \in I$, the set $E_{\alpha}:=\left\{x \in C \mid x_{\alpha} \in \operatorname{clB}_{\alpha}(x)\right\}$ is closed in $C$, since $\operatorname{clB}_{\alpha}$ is upper semicontinuous by (3). Define a set-valued mapping $Q_{\alpha}: C \longrightarrow 2^{C_{\alpha}}$ by

$$
Q_{\alpha}(x):= \begin{cases}\left(A_{\alpha} \cap P_{\alpha}\right)(x), & \text { if } x \in E_{\alpha} \\ H-\operatorname{co} A_{\alpha}(x), & \text { if } x \notin E_{\alpha} .\end{cases}
$$


We shall show that all the hypotheses of Lemma 3 hold to the family $\left\{Q_{\alpha} \mid \alpha \in I\right\}$. First, for each $\alpha \in I$, the set

$$
\begin{aligned}
\left\{x \in C \mid Q_{\alpha}(x)\right\} & =\left\{x \in E_{\alpha} \mid Q_{\alpha}(x) \neq \varnothing\right\} \cup\left\{x \in C \backslash E_{\alpha} \mid Q_{\alpha}(x) \neq \varnothing\right\} \\
& =\left\{x \in E_{\alpha} \mid\left(A_{\alpha} \cap P_{\alpha}\right)(x) \neq \varnothing\right\} \cup\left(C \backslash E_{\alpha}\right) \\
& =\left(E_{\alpha} \cap D_{\alpha}\right) \cup\left(C \backslash E_{\alpha}\right) \\
& =D_{\alpha} \cup\left(C \backslash E_{\alpha}\right)
\end{aligned}
$$

is open in $C$ by (5).

Furthermore, for $x \in C$ with $Q_{\alpha}(x) \neq \varnothing$, we consider the following two cases. In case $x \notin E_{\alpha}$, we take $T_{\alpha}=H$-co $A_{\alpha}$. Then, $Q_{\alpha}(z) \subset T_{\alpha}(z)$ for all $z \in C$; and $T_{\alpha}^{-1}(y)$ is open in $C$ for each $y \in C_{\alpha}$, by using (2) and Lemma 1. Notice that the set $N_{\alpha}:=C \backslash E_{\alpha}$ is an open neighborhood of $x$ in $C$. Furthermore, for all $z \in N_{\alpha}, z \notin E_{\alpha}$, and hence $z_{\alpha} \notin c l B_{\alpha}(z)$. This implies that $\pi_{\alpha}(z)=z_{\alpha} \notin H$-coT $T_{\alpha}(z)$, by (1). Therefore, $T_{\alpha}$ is an $\mathcal{L}_{\pi_{\alpha}}$-majorant of $Q_{\alpha}$ at $x$.

On the other hand, if $x \in E_{\alpha}$, then $Q_{\alpha}(x)=\left(A_{\alpha} \cap P_{\alpha}\right)(x)$. By (4), there exists an $\mathcal{L}_{\pi_{\alpha}}$-majorant $S_{\alpha}$ of $A_{\alpha} \cap P_{\alpha}$ at $x$; that is, there is an open neighborhood $N_{\alpha}$ of $x$ in $C$ such that

(a) $\left(A_{\alpha} \cap P_{\alpha}\right)(z) \subset S_{\alpha}(z)$ for each $z \in C$;

(b) $\pi_{\alpha}(z) \notin H-\cos \alpha(z)$ for each $z \in N_{\alpha}$;

(c) $S_{\alpha}^{-1}(y)$ is open in $C$ for all $y \in C_{\alpha}$.

By (5), we may assume that $N_{\alpha} \subset D_{\alpha}$, so that $A_{\alpha}(z) \cap P_{\alpha}(z) \neq \varnothing$ for all $z \in N_{\alpha}$. Now, we define $T_{\alpha}: C \longrightarrow 2^{C_{\alpha}}$ by

$$
T_{\alpha}(z):= \begin{cases}H-\operatorname{co} A_{\alpha}(z) \cap S_{\alpha}(z), & \text { if } z \in E_{\alpha}, \\ H-c o A_{\alpha}(z), & \text { if } z \notin E_{\alpha} .\end{cases}
$$

By (a), we see $Q_{\alpha}(z) \subset T_{\alpha}(z)$ for each $z \in C$. In addition, $\pi_{\alpha}(z) \notin H$-co $T_{\alpha}(z)$ for each $z \in N_{\alpha}$, by (1) and (b). Moreover, for each $y \in C_{\alpha}$, the set

$$
\begin{aligned}
T_{\alpha}^{-1}(y) & =\left\{z \in C \mid y \in T_{\alpha}(z)\right\} \\
& =\left\{z \in E_{\alpha} \mid y \in T_{\alpha}(z)\right\} \cup\left\{z \in C \backslash E_{\alpha} \mid y \in T_{\alpha}(z)\right\} \\
& =\left\{z \in E_{\alpha} \mid y \in H-c o A_{\alpha}(z) \cap S_{\alpha}(z)\right\} \cup\left\{z \in C \backslash E_{\alpha} \mid y \in H-c o A_{\alpha}(z)\right\} \\
& =\left(E_{\alpha} \cap\left(H-c o A_{\alpha}\right)^{-1}(y) \cap S_{\alpha}^{-1}(y)\right) \cup\left(\left(C \backslash E_{\alpha}\right) \cap\left(H-c o A_{\alpha}\right)^{-1}(y)\right) \\
& =\left(S_{\alpha}^{-1}(y) \cup\left(C \backslash E_{\alpha}\right)\right) \cap\left(H-c o A_{\alpha}\right)^{-1}(y)
\end{aligned}
$$

is open in $C$, by (c) and Lemma 1. This shows that $T_{\alpha}$ is an $\mathcal{L}_{\pi_{\alpha}}$-majorant of $Q_{\alpha}$ at $x$. Accordingly, we conclude that $Q_{\alpha}$ is $\mathcal{L}_{\pi_{\alpha}}$-majorized. In addition, for each $\alpha \in I$, by assumption (6), there is $y_{\alpha} \in K_{\alpha}$ such that $y_{\alpha} \in H-c o\left(A_{\alpha} \cap P_{\alpha}\right)(x) \subset H-c o Q_{\alpha}(x)$ for all $x \in C \backslash K$. It follows from Lemma 3 that there exists $\hat{x} \in K$ such that $Q_{\alpha}(\hat{x})=\varnothing$. Since $A_{\alpha}(\hat{x}) \neq \varnothing$ by (1), we have $\hat{x} \in E_{\alpha}$, and hence $\hat{x}_{\alpha} \in c l B_{\alpha}(\hat{x})$ and $A_{\alpha}(\hat{x}) \cap P_{\alpha}(\hat{x})=Q_{\alpha}(\hat{x})=\varnothing$; that is, $\hat{x}$ is an equilibrium of $\Omega$.

Remark 3. Theorem 3 generalizes Yannelis and Prabhakar [6], Kim [24], and Yuan and Tarafdar [9] in several ways:

(1) We focus on the setting of general H-spaces without any linear or convex structure;

(2) The set I of agents can be any infinite set;

(3) The strategy set $C_{\alpha}$ need not be compact or metrizable;

(4) The preference correspondence $P_{\alpha}$ need not be of class $\mathcal{L}$, and does not require the usual lower semicontinuous assumptions, such as the earlier works [20] (Theorem 3 and its Corollary) and [9] (Theorem 6.1). 
Here, we can construct an applicable example, so that the abstract economy $\Omega$ still has an equilibrium point, even though the correspondences are not lower semicontinuous and the strategy sets are not compact.

Example 1. Let the strategy set $C_{\alpha}=[0, \infty)$, with $\alpha \in I=\{1,2\}, Q$ denote the set of rational numbers, and the correspondences $A_{\alpha}, B_{\alpha}, P_{\alpha}: C_{1} \times C_{2} \longrightarrow 2^{C_{\alpha}}$ be defined as follows:

$$
\begin{aligned}
& A_{1}\left(x_{1}, x_{2}\right):=\left\{\begin{array}{cc}
(0,1), & \text { if } 0 \leq x_{1}<x_{2}<1 \text { and } x_{1} \notin Q \text { or } x_{2} \notin Q \\
\left(\frac{x_{1}+x_{2}}{2}, 1\right), & \text { if } 0 \leq x_{1}<x_{2}<1 \text { and } x_{1}, x_{2} \in Q \\
\{0\}, & \text { if } 0 \leq x_{2} \leq x_{1}<1 \\
(0,2), & \text { if } x_{1} \geq 1 \text { or } x_{2} \geq 1
\end{array},\right. \\
& A_{2}\left(x_{1}, x_{2}\right):=\left\{\begin{array}{cc}
(0,1), & \text { if } 0 \leq x_{2}<x_{1}<1 \text { and } x_{1} \notin Q \text { or } x_{2} \notin Q \\
\left(\frac{x_{1}+x_{2}}{2}, 1\right), & \text { if } 0 \leq x_{2}<x_{1}<1 \text { and } x_{1}, x_{2} \in Q \\
\{0\}, & \text { if } 0 \leq x_{1} \leq x_{2}<1 \\
(0,2), & \text { if } x_{1} \geq 1 \text { or } x_{2} \geq 1
\end{array}\right.
\end{aligned}
$$

whereas $B_{1}\left(x_{1}, x_{2}\right)=B_{2}\left(x_{1}, x_{2}\right)=[0, \infty)$ for each $\left(x_{1}, x_{2}\right) \in C_{1} \times C_{2}$, and

$$
\begin{gathered}
P_{1}\left(x_{1}, x_{2}\right):=\left\{\begin{array}{cc}
\left(\frac{x_{1}+x_{2}}{2}, 1\right), & \text { if } 0 \leq x_{1}<x_{2}<1 \text { and } x_{1} \notin Q \text { or } x_{2} \notin Q \\
(0,1), & \text { if } 0 \leq x_{1}<x_{2}<1 \text { and } x_{1}, x_{2} \in Q \\
\varnothing, & \text { if }\left(x_{1}, x_{2}\right) \in S \\
\{0\}, & \text { if } 0 \leq x_{2}<x_{1}<1 \\
\{1\}, & \text { if } x_{1}>1 \text { or } x_{2}>1
\end{array},\right. \\
P_{2}\left(x_{1}, x_{2}\right):=\left\{\begin{array}{cc}
\left(\frac{x_{1}+x_{2}}{2}, 1\right), & \text { if } 0 \leq x_{2}<x_{1}<1 \text { and } x_{1} \notin Q \text { or } x_{2} \notin Q \\
(0,1), & \text { if } 0 \leq x_{2}<x_{1}<1 \text { and } x_{1}, x_{2} \in Q \\
\varnothing, & \text { if }\left(x_{1}, x_{2}\right) \in S \\
\{0\}, & \text { if } 0 \leq x_{1}<x_{2}<1 \\
\{1\}, & \text { if } x_{1}>1 \text { or } x_{2}>1
\end{array} .\right.
\end{gathered}
$$

Here, the set $S:=\left\{\left(x_{1}, x_{2}\right) \mid x_{1}=x_{2}\right.$ or $x_{1}=1$ or $\left.x_{2}=1\right\} \cap([0,1] \times[0,1])$. Then, $A_{1}$ is not lower semicontinuous at $\left(\frac{1}{2}, \frac{1}{\sqrt{3}}\right)$ and $A_{2}$ is not lower semicontinuous at $\left(\frac{1}{\sqrt{3}}, \frac{1}{2}\right)$. Similarly, both $P_{1}$ and $P_{2}$ are not lower semicontinuous. It is easy to check that each set $D_{\alpha}$ in Theorem 3 is open in $C:=C_{1} \times C_{2}$, since it is the complement of the closed set $S$. In addition, $A_{\alpha} \cap P_{\alpha}$ is an $\mathcal{L}_{\pi_{\alpha}}$-majorant of itself by the fact $\pi_{\alpha}\left(x_{1}, x_{2}\right)=x_{\alpha} \notin H-c o\left(A_{\alpha}\left(x_{1}, x_{2}\right) \cap P_{\alpha}\left(x_{1}, x_{2}\right)\right)$ for each $\alpha=1$, 2 . Furthermore, the coercive condition holds clearly by taking $K:=[0,1] \times[0,1]$ and $y=(1,1)$.

An $H$-space $X$ is called an l.c.-space, if $X$ is an uniform space with uniformity $\mathcal{U}$ having a base $\mathcal{B}$ of symmetric entourages such that, for each $V \in \mathcal{B}$, the set $V(E):=\{y \in X \mid(x, y) \in V$ for some $x \in E\}$ is $H$-convex whenever $E$ is $H$-convex. In the setting of $l$.c.-spaces, we now establish a new fixed point theorem as follows:

Theorem 4. Let $C$ be an H-convex subset of an l.c.-space $X$, and $K$ be a nonempty compact subset of $C$. Suppose that $T: C \longrightarrow 2^{C}$ is a continuous set-valued mapping with nonempty values and $P: C \longrightarrow 2^{C}$ is a set-valued mapping such that

(1) the mapping $T \cap P: C \longrightarrow 2^{C}$ is $\mathcal{L}$-majorized;

(2) the set $D:=\{x \in C \mid T(x) \cap P(x) \neq \varnothing\}$ is open in $C$;

(3) there exists $y \in K$ such that $C \backslash K \subset(H-c o(T \cap P))^{-1}(y)$.

Then, the mapping $x \mapsto c l(H-c o T(x))$ has a fixed point $\hat{x} \in C$ such that $T(\hat{x}) \cap P(\hat{x})=\varnothing$. Moreover, if each image $T(x)$ is closed and H-convex, then $\hat{x}$ is a fixed point of $T$. 
Proof. Define two mappings $A=T$ and $B=H$-coT. Notice that $A$ has open lower sections, since $T$ is lower semicontinuous. In addition, since $T$ is upper semicontinuous, $c l B$ is also upper semicontinuous, due to Tarafdar and Watson [30]. In addition, by (3), we have $y \in H-c o(T \cap P)(x)$ for all $x \in C \backslash K$. Thus, applying Theorem 3, we have an equilibrium $\hat{x}$ of the abstract economy $\Omega:=(C, A, B, P)$. That is, $\hat{x} \in \operatorname{cl} B(\hat{x})$, and $A(\hat{x}) \cap P(\hat{x})=\varnothing$. Thus, $\hat{x}$ is a fixed point of the mapping $x \mapsto c l(H-\operatorname{coT}(x))$, and $T(\hat{x}) \cap P(\hat{x})=\varnothing$. Moreover, when $T$ has closed and $H$-convex values, $\hat{x} \in \operatorname{clB}(\hat{x})=\operatorname{cl}(H-\operatorname{coT}(\hat{x}))=T(\hat{x})$, and hence $\hat{x}$ becomes a fixed point of $T$.

As a consequence of Theorem 4, we have a version of Tarafdar fixed point theorem [31] (Theorem 2.1) as follows.

Corollary 1. Let $C$ be an H-convex subset of an l.c.-space $X$, and $K$ be a nonempty compact subset of $C$. If $T: C \longrightarrow 2^{C}$ is a continuous set-valued mapping with nonempty and closed H-convex values such that $C \backslash K \subset T^{-1}(y)$ for some $y \in K$, then $T$ has a fixed point in $C$.

Proof. Assume that $T$ has no fixed point. Then, $x \notin T(x)=H$-coT $(x)$ for each $x \in C$. In addition, since $T$ is lower semicontinuous, $T^{-1}(x)$ is open for each $x \in C$. Thus, $T$ is of class $\mathcal{L}$, and hence $T$ is $\mathcal{L}$-majorized. Define the set-valued mapping $P: C \longrightarrow 2^{C}$ by $P(x)=C$ for all $x \in C$, so that the set $D:=\{x \in C \mid T(x) \cap P(x) \neq \varnothing\}=C$ is open in $C$. Furthermore, it follows that $C \backslash K \subset T^{-1}(y)=(H-c o(T \cap P))^{-1}(y)$. Applying Theorem 4, we conclude that $T$ has a fixed point in $C$. This contradicts with our assumption, and therefore the proof is complete.

It is known that most fixed point theorems often require the strategy set to be compact; however, Corollary 1 involves a kind of coercive condition to avoid such a constraint qualification. We can give a simple example here.

Example 2. Let the set-valued mapping $T: C \longrightarrow 2^{C}$, where $C=(0, \infty)$, be defined by

$$
T(x):= \begin{cases}\left\{y \in C \mid 0<y \leq x^{2}\right\}, & \text { if } x \in(0,1], \\ (0,1], & \text { if } x \in(1, \infty) .\end{cases}
$$

Then, $T$ is continuous on $C$ and satisfies the coercive condition in Corollary 1 by taking the compact set $K:=(0,1]$ and $y=1$. Thus, $T$ admits a fixed point.

\section{Results on Eigenvector Problems}

In what follows, $\mathcal{K}$ will denote the field of real numbers $\mathbb{R}$ or the field of complex numbers $\mathbb{C}$. Let $(E,\|\cdot\|)$ be a normed linear space with origin $\theta$. Let $\Re: E \longrightarrow 2^{E}$ be a set-valued mapping defined by

$$
\Re(v):=\{r v \mid r \in \mathcal{K}\}, v \in E .
$$

It is obvious that $\Re(\theta)=\{\theta\}$ and $\Re(v)$ is closed and convex for each $v \in E$. Let $C$ be a nonempty subset of $E$ and $f: C \longrightarrow E$ be a mapping. The kernel of $f$ is the set ker $f$ denoted by

$$
\operatorname{ker} f:=\{x \in C \mid f(x)=\theta\} .
$$

A scalar $\lambda \in \mathcal{K}$ and a point $v \in C$ with $v \neq \theta$ are called an eigenvalue and the corresponding eigenvector of $f$, respectively, if $f(v)=\lambda v$. In this section, we study the following eigenvector problem (EIVP, for short):

(EIVP) Find $v \in C$ with $v \neq \theta$ and $\lambda \in \mathcal{K}$ such that $f(v)=\lambda v$.

The following result can be proved immediately from definitions. 
Lemma 4. Let $(E,\|\cdot\|)$ be a normed linear space and $C$ be a nonempty subset of $E$. Let the function $\mu: E \longrightarrow \mathbb{R}$ be defined by

$$
\mu(x)=d(x, C):=\inf \{\|x-a\| \mid a \in C\}, x \in E .
$$

Then, $\mu$ is continuous. Furthermore, if $C$ is convex, then $\mu$ is convex.

The following lemma is very crucial for our results.

Lemma 5. Let $(E,\|\cdot\|)$ be a normed linear space with origin $\theta, C$ be a nonempty convex subset of $E, \Re(x):=$ $\{r x \mid r \in \mathcal{K}\}$ for $x \in C$, and $f: C \longrightarrow E$ be a continuous mapping. Then, for any $y \in C$, the set

$$
\{x \in C \mid d(f(y), \Re(x)) \geq d(f(x), \Re(x))\}
$$

is a nonempty closed subset of $C$.

Proof. Let $y \in C$ be fixed. Clearly, $y \in\{x \in C \mid d(f(y), \Re(x)) \geq d(f(x), \Re(x))\} \neq \varnothing$. Following a similar argument as in the proof of [32] (Theorem 3.2), we can prove its closedness.

By applying Theorem 1, we give a sufficient condition for the existence of the solution of eigenvector problem.

Theorem 5. Let $(E,\|\cdot\|)$ be a normed linear space with origin $\theta, C$ be a nonempty convex subset of $E$ with $\theta \notin C$, and $f: C \longrightarrow E$ be an affine and continuous mapping satisfying $\operatorname{ker} f \neq \varnothing$. Suppose that

(H) there exists a nonempty compact subset $K$ of $C$ such that for each $x \in C \backslash K$ there exists $y \in K$ such that $d(f(y), \Re(x))<d(f(x), \Re(x))$.

Then, the problem (EIVP) has a solution; that is, there exists $v \in C$ with $v \neq \theta$ and $\lambda \in \mathcal{K}$ such that $f(v)=\lambda v$.

Proof. Let $\kappa: C \times C \longrightarrow \mathbb{R}$ be defined by

$$
\kappa(x, y):=d(f(y), \Re(x))-d(f(x), \Re(x)) .
$$

For each $x \in C$, since $f$ is affine and continuous, by Lemma 4 , the function $y \longrightarrow \kappa(x, y)$ is convex and continuous. Define $T: C \longrightarrow 2^{C}$ by

$$
T(x):=\{y \in C \mid \kappa(x, y)<0\}, x \in C .
$$

Thus, $T(x)$ is convex for each $x \in C$. Clearly, $x \notin T(x)=\operatorname{coT}(x)$ for each $x \in C$. For each $y \in C$, by Lemma 5 , we know that

$$
T^{-1}(y)=\{x \in C \mid \kappa(x, y)<0\}
$$

is open in $C$. Hence, $T$ is a set-valued mapping of class $\mathcal{L}$. By our coercivity condition $(\mathrm{H})$, there exists a nonempty compact subset $K$ of $C$ such that $K \cap T(x) \neq \varnothing$ for each $x \in C \backslash K$. Applying Theorem 1 , there exists $v \in C$ such that $T(v)=\varnothing$, or, equivalently,

$$
d(f(x), \Re(v)) \geq d(f(v), \Re(v)) \text { for all } x \in C .
$$

Clearly, $v \neq \theta$ due to $\theta \notin C$. Since $\operatorname{ker} f \neq \varnothing$, there exists $z \in C$ such that $f(z)=\theta$. Hence, we get

$$
d(f(v), \Re(v)) \leq d(f(z), \Re(v))=0,
$$

which deduces $d(f(v), \Re(v))=0$. By the closedness of $\Re(v)$, we obtain $f(v) \in \Re(v)$. Therefore, there exists $\lambda \in \mathcal{K}$ such that $f(v)=\lambda v$. 
Remark 4. It is worth mentioning that the coercivity condition $(H)$ in Theorem 5 is different from the two main coercivity conditions $\left(H_{1}\right)$ in [32] (Theorem 3.4) and $\left(H_{2}\right)$ in [32] (Theorem 4.1). Thus, Theorem 5 is not a special case of any result in [32].

The following conclusion is immediate from Theorem 5.

Corollary 2. Let $(E,\|\cdot\|)$ be a normed linear space with origin $\theta, C$ be a nonempty compact convex subset of $E$ with $\theta \notin C$, and $f: C \longrightarrow E$ be an affine and continuous mapping satisfying ker $f \neq \varnothing$. Then, the problem (EIVP) has a solution.

Here, we give an example illustrating Theorem 5 (or Corollary 2).

Example 3. Let $E=\mathbb{R}^{3}$ with the usual metric and $\mathcal{K}=\mathbb{R}$. Then, $C:=\left\{(0, y, z) \in \mathbb{R}^{3} \mid 1 \leq y \leq 5,-6 \leq\right.$ $z \leq 9\}$ is a nonempty compact convex subset of $E$. Clearly, $\theta:=(0,0,0) \notin C$. Define $f: C \longrightarrow E$ by

$$
f(x, y, z):=(x, 2 y+z, 2 y+z) \text {. }
$$

Then, $f$ is affine and continuous. Let $\hat{\lambda}:=3$ and $\hat{v}:=(0,1,1) \in C$. It is easy to see that $f(\hat{v})=\hat{\lambda} \hat{v}$, so $(\hat{\lambda}, \hat{v})$ is a solution of problem (EIVP). On the other hand, since $(0,1,-2) \in C$ and $f(0,1,-2)=(0,0,0)$, we have $\operatorname{ker} f \neq \varnothing$. Hence, we can also show that the problem (EIVP) has a solution by using Theorem 5 (or Corollary 2).

\section{Conclusions}

In this paper, we prove two existence theorems of maximal elements in noncompact $H$-spaces. More accurately, we deal with the correspondence to be of $\mathcal{L}$-majorized mappings in the setting of noncompact strategy sets, but merely require some milder coercive conditions. As applications, we obtain an equilibrium existence theorem for general abstract economies, together with a new fixed point theorem, and give a sufficient condition for the existence of solutions of the eigenvector problem (EIVP). More precisely, we make the following remarks, so that the reader understands what we consider to be.

(1) In order to prove our results, we introduce a new concept of $H$-spaces with covering polytopes, and develop some technical tools. It is known that the mapping $H$-coT of a set-valued mapping $T$ in any locally convex topological vector space preserves the open lower sections. Lemma 1 indicates that such a property still holds in any $H$-space with covering polytopes. The reader might study further some general topological space, such as G-spaces [27] and FC-spaces [33], and offers some interesting property to avoid such an additional assumption.

(2) Many existence theorems of maximal elements do not need any compactness on the strategy sets. In the literature, there are many ways to control the noncompact case. One classical way is to consider various set-valued mappings, such as compact mapping or condensing mapping. The other way is to offer a proper and novel coercive condition on the strategy set, such as [25] (Theorem 4), [24] (Theorem 3), [34] (Theorem 3.3 and its corollary), and [35] (Theorem 3 and its corollary), which are closely related to our coercive conditions.

(3) KKM theory and fixed point theorem are often related, and play crucial roles in proving the existence of maximal elements. They are logically equivalent with various optimization problems, such as minimax inequality, variational inequality, coincidence theorem, complementarity problems, and equilibrium problems. As we know, most of the earlier works present existence theorems of maximal elements by using fixed point theorems. However, we have an interesting tour in this paper. In fact, we apply a general KKM theorem (see Lemma 2) of Chang and Ma [13] (Theorem 1) to establish our existence theorems of maximal elements, and then obtain a general fixed point theorem. More results on KKM theory can be found in [27,28,30,31,33]. 
Author Contributions: The authors contributed equally to this work. Both authors read and approved the final manuscript.

Funding: The first author is partially supported by the Ministry of Science and Technology of the Republic of China. The second author is supported by Grant No. MOST 107-2115-M-017-004-MY2 of the Ministry of Science and Technology of the Republic of China.

Acknowledgments: The authors wish to express their hearty thanks to the anonymous referees for their valuable suggestions and comments.

Conflicts of Interest: The authors declare no conflict of interest.

\section{References}

1. Arrow, K.J.; Debreu, G. Existence of an equilibrium for a competitive economiy. Econometrica 1954, 22, $265-290$. [CrossRef]

2. Debreu, G. A social equilibrium existence theorem. Proc. Natl. Acad. Sci. USA 1952, 38, 886-893. [CrossRef] [PubMed]

3. Shafer, W.; Sonnenschein, H. Equilibrium in abstract economies without ordered preferences. J. Math. Econ. 1975, 2, 345-348. [CrossRef]

4. Borglin, A.; Keiding, H. Existence of equilibrium actions and of equilibrium: A note on the "new" existence theorems. J. Math. Econ. 1976, 3, 313-316. [CrossRef]

5. Gale, D.; Mas-Colell, A. On the role of complete, translative preferences in equilibrium theory. In Equilibrium and Disequilibrium in Economies Theory; Schwodiauer, G., Ed.; Dordrecht Reidel: Boston, MA, USA, 1978; pp. 7-14.

6. Yannelis, N.C.; Prabhakar, N.D. Existence of maximal elements and equilibria in linear topological spaces. J. Math. Econ. 1983, 12, 233-245. [CrossRef]

7. Ding, X.P.; Tan, K.K. Matching theorems, fixed point theorems and minimax inequalities. J. Aust. Math. Soc. 1990, 49, 111-128.

8. Tarafdar, E. Fixed point theorems in H-spaces and equilibrium points of abstract economies. J. Aust. Math. Soc. 1992, 53, 252-260. [CrossRef]

9. Yuan, G.X.Z.; Tarafdar, E. Maximal elements and equilibria of generalized games for $\mathcal{U}$-majorized and condensing correspondences. Int. J. Math. Sci. 1999, 22, 179-189. [CrossRef]

10. Park, S. Remarks on topologies of generalized convex spaces. Nonlinear Funct. Anal. Appl. Math. 2000, 5, 67-79.

11. He, W.; Yannelis, N.C. Equilibria with discontinuous preferences: New fixed point theorems. J. Math. Anal. Appl. 2017, 450, 1421-1433. [CrossRef]

12. Chang, S.Y. On the Nash equibrium. Soochow J. Math. 1990, 16, 241-248.

13. Chang, S.S.; Ma, Y.H. Generalized KKM theorem on $H$-space with applications. J. Math. Anal. Appl. 1992, 163, 406-421. [CrossRef]

14. Huang, C.-H.; Chu, L.-J. A fixed point theorem with application to abstract economy. J. Nonlinear Conv. Anal. 2016, 17, 177-184.

15. Chu, L.-J.; Huang, C.-H. A common maximal element of condensing mappings. Fixed Point Theory 2019, 20, Available online: http:/ / www.math.ubbcluj.ro/ nodeacj/sfptcj.html (accessed on 1 March 2019).

16. Du, Y.M. Fixed points, maximal elements and equilibria of generalized games in abstract convex spaces. Abstr. Appl. Anal. 2012, 2012, 842515. [CrossRef]

17. Lin, L.-J.; Ansari, Q.H. Collective fixed points and maximal elements with applications to abstract economies. J. Math. Anal. Appl. 2004, 296, 455-472. [CrossRef]

18. $\mathrm{Wu}, \mathrm{X}$. Existence theorem for maximal elements in $\mathrm{H}$-spaces with applications on the minimax inequalities and equilibrium of games. J. Appl. Anal. 2000, 6, 283-293. [CrossRef]

19. Wu, X.; Shen, Z.F. Equilibrium of abstract economy and generalized quasi-variational inequality in $\mathrm{H}$-spaces. Topol. Appl. 2005, 153, 123-132. [CrossRef]

20. Wu, X.; Yuan, G.X.Z. On equilibrium problem of abstract economy, generalized quasi-variational inequality, and an optimization problem in locally H-spaces. J. Math. Appl. Anal. 2003, 282, 495-504. [CrossRef]

21. Wu, Y.-L.; Huang, C.-H.; Chu, L.-J. An extension of Mehta Theorem with applications. J. Math. Anal. Appl. 2012, 391, 489-495. [CrossRef] 
22. Bardaro, C.; Ceppitelli, R. Some further generalizations of Knaster Kuratowski Mazurkiewicz theorem and minimax inequalities. J. Math. Anal. Appl. 1988, 132, 484-490. [CrossRef]

23. Guo, W.; Cho, Y.J. A new maximal element theorem in H-space with applications. Nonlinear Anal. 2008, 68, 2194-2203. [CrossRef]

24. Kim, W.K. Existence of maximal element and equilibrium for a nonparacompact N-person game. Proc. Am. Math. Soc. 1992, 116, 797-807.

25. Ding, X.P.; Kim, W.K.; Tan, K.K. Equilibria of non-compact generalized games with $L^{*}$-majorized preference correspondences theorems. J. Math. Anal. Appl. 1992, 164, 508-517. [CrossRef]

26. Tarafdar, E. A fixed point theorem in H-spaces and related results. Bull. Aust. Math. Soc. 1990, 42, $133-140$. [CrossRef]

27. Park, S. Elements of the KKM theory for generalized convex spaces. Korean J. Comput. Appl. Math. 2000, 7, 1-28.

28. Wen, K.T. A new KKM theorem in L-convex spaces and some applications. Comput. Math. Appl. 2008, 56, 2781-2785. [CrossRef]

29. Fan, K. Some properties of convex sets related to fixed point theorem. Math. Ann. 1984, 266, 519-537. [CrossRef]

30. Tarafdar, E.; Watson, P.J. Coincidence and the Fan-Glicksberg Fixed Point Theorem in Locally H-Convex Uniform Spaces; Research Report; The University of Queensland: Brisbane, Australia, 1997.

31. Tarafdar, E. Fixed point theorem in locally H-convex uniform spaces. Nonlinear Anal. 1997, 29, 971-978. [CrossRef]

32. Lin, L.-J.; Du, W.-S. On common quasi-eigenvector problems. Nonlinear Anal. 2008, 69, 463-471. [CrossRef]

33. Ding, X.P. Maximal elements of the $G_{K K M}$-mappings in product $F C$-spaces and applications (I). Nonlinear Anal. 2007, 67, 963-973. [CrossRef]

34. Yuan, G.X.Z.; Isac, G.; Tan, K.K.; Yu, J. The study of maximal inequalities, abstract economies and applications to variational inequalities and Nash equilibria. Acta Appl. Math. 1998, 54, 135-166. [CrossRef]

35. Chang, S.Y. Maximal elements in noncompact spaces with application to equilibria. Proc. Am. Math. Soc. 2003, 132, 535-541. [CrossRef]

(C) 2019 by the authors. Licensee MDPI, Basel, Switzerland. This article is an open access article distributed under the terms and conditions of the Creative Commons Attribution (CC BY) license (http://creativecommons.org/licenses/by/4.0/). 